\title{
Prevalencia de anticuerpos neutralizantes contra los serotipos del virus dengue en universitarios de Tabasco, México
}

\author{
Gilma Guadalupe Sánchez-Burgos, Dr en C, ${ }^{(1,2)}$, Miguel Ángel López-Alvarado, M en C, ${ }^{(2)}$ \\ Deyanira Castañeda-Desales, Biol, ${ }^{(3)}$ Juan Ruiz-Gómez, MC, ${ }^{(3) \dagger}$ José Ramos-Castañeda, M en C. ${ }^{(3)}$
}

\section{Sánchez-Burgos GG, López-Alvarado MA Castañeda-Desales D, Ruiz-Gómez J, ${ }^{\dagger}$ Ramos-Castañeda J. Prevalencia de anticuerpos neutralizantes contra los serotipos del virus dengue en universitarios de Tabasco, México. \\ Salud Publica Mex 2008;50:362-366.}

\section{Resumen}

Objetivo. Determinar la seroprevalencia de anticuerpos neutralizantes de los serotipos del virus dengue en estudiantes universitarios de Tabasco, México, durante los meses de septiembre a noviembre del año 2005. Material y métodos. Se determinó la presencia de lgG contra el virus en el suero de estudiantes que acudieron al centro clínico de la universidad; en los sueros positivos se determinaron los anticuerpos neutralizantes mediante el ensayo de reducción de placa lítica. Resultados. La prevalencia de lgG contra el dengue fue de 9.1\%; de esta proporción, los anticuerpos neutralizantes fueron DENV-I (20\%), DENV-2 (I00\%), DENV-3 (4\%) y DENV-4 $(68 \%)$. Conclusiones. Este estudio muestra que el serotipo transmitido con mayor frecuencia en el estado de Tabasco es el DENV-2, aunque no ha sido el aislado con más frecuencia. La elevada prevalencia de anticuerpos neutralizantes contra el DENV-4, al parecer de reacción cruzada, podría explicar la baja circulación de este serotipo en Tabasco.

Palabras clave: dengue; anticuerpos; transmisión; México

\author{
Sánchez-Burgos GG, López-Alvarado MA, \\ Castañeda-Desales D, Ruiz-Gómez J, ${ }^{\dagger}$ Ramos-Castañeda J. \\ Prevalence of neutralizing antibodies \\ to dengue virus serotypes \\ in university students from Tabasco, Mexico. \\ Salud Publica Mex 2008;50:362-366.
}

\begin{abstract}
Objective. Determine the seroprevalence of neutralizing antibodies to dengue virus in students from the state university of Tabasco, Mexico. Material and Methods. A transversal study was conducted of serum collected from students between September and November, 2005. The sera were screened for anti-dengue IgG and those that had evidence of dengue antibodies were analyzed by a plaque reduction neutralization test. Results. Prevalence of anti-dengue lgG was $9.1 \%$. The frequency of neutralizing antibodies was $100 \%$ for DENV-2, 68\% for DENV-4, 20\% for DENV-I, and $4 \%$ for DENV-3. Conclusions. We found that in this population, DENV-2 circulates more than DENV-3 despite the fact that DENV-3 is more frequently isolated. Unexpectedly, neutralizing antibodies against DENV-4 were frequently found even though this serotype is almost extinct; thus, it is probable that cross-immunity could suppress DEN-4 transmission, as has been suggested.
\end{abstract}

Key words: dengue; antibodies; viral transmission; Mexico

(I) Unidad de Investigación Médica, IMSS. Mérida, Yucatán.

(2) División Académica de Ciencias de la Salud, Universidad Juárez Autónoma de Tabasco.Villahermosa, Tabasco.

(3) Centro de Investigación sobre Enfermedades Infecciosas, INSP. Cuernavaca, Morelos, México.

Fecha de recibido: 19 de septiembre de 2007 - Fecha de aceptado: 15 de abril de 2008 Solicitud de sobretiros: Mtro. José Ramos Castañeda. Centro de Investigación sobre Enfermedades Infecciosas, INSP. Av. Universidad 655, col. Santa María Ahuacatitlán. 62508 Cuernavaca, Morelos, México. Correo electrónico: jcastane@insp.mx 
$E^{1}$ dengue es la enfermedad viral transmitida por vector más prevalente en el mundo ${ }^{1}$ y constituye un reto de la salud pública ya que no se dispone de una vacuna y las medidas de control del vector sólo tienen un carácter reactivo; ${ }^{2}$ en este sentido, el costo de los brotes del dengue se ha calculado en casi 465 DALY (años de vida ajustados y perdidos por incapacidad, por sus siglas en inglés), sin contar el efecto que tienen en sectores económicamente activos como los de servicio y turismo. ${ }^{3}$ En consecuencia, es importante contar con un sistema que permita reducir la incidencia y racionalizar al mismo tiempo los recursos y enfocarlos en áreas de mayor probabilidad de presentación de brotes.

Uno de los elementos que debe considerar este sistema es la reacción inmunitaria de la población a la infección, ya que se ha relacionado una mayor incidencia de casos graves de fiebre hemorrágica por dengue/ síndrome de choque por dengue (FHD/SCD) con la respuesta inmunitaria heterotípica no neutralizante, ${ }^{2}$ aunque un estudio reciente demuestra que este tipo de inmunidad no contribuye a la transmisión en el sureste asiático (SEA). ${ }^{4}$ Otro aspecto de la inmunidad contra el virus dengue que debe evaluarse es la reacción inmunitaria heterotípica neutralizante, toda vez que este tipo de inmunidad parece dirigir el patrón de transmisión. ${ }^{5}$

Casi sin excepción, los modelos epidemiológicos disponibles se basan en el comportamiento de la transmisión del dengue en el SEA; empero, hay diferencias significativas entre esa región y los países americanos; por ejemplo, mientras que en el SEA los niños son la población más afectada, en las naciones americanas todos los grupos etarios se afectan; en el SEA prácticamente no hay casos de FHD porque casi todos desarrollan muy rápido el $\mathrm{SCD}$, mientras que en América casi todos los casos corresponden a FHD, con escasos casos de SCD; esto refleja una tasa de mortalidad relativamente baja que se presenta en los países americanos en comparación con el SEA. ${ }^{6}$

Son pocos los estudios sobre la inmunidad contra el virus del dengue en las naciones latinoamericanas y, en particular, en México son aún menos. En fecha reciente, un estudio en una población del estado de Veracruz demostró que la prevalencia de anticuerpos IgG contra el virus del dengue alcanzó casi $80 \%,{ }^{7}$ otro estudio, éste en la población del estado de Yucatán, reveló que en la muestra seleccionada la seroprevalencia de anticuerpos inhibidores de la hemaglutinación $(\mathrm{IH})$ fue de $72.5 \%{ }^{8}$ Sin embargo, como ya se mencionó, es necesario conocer el tipo de inmunidad presente en una población para evaluar la vulnerabilidad de ésta a presentar brotes de dengue. En este trabajo se evaluó la prevalencia de anticuerpos neutralizantes contra el dengue en una muestra de estudiantes de la Universidad Juárez Autónoma de Tabasco (UJAT) en 2005.

\section{Material y métodos}

Se realizó un estudio transversal para determinar el nivel de anticuerpos neutralizantes contra los cuatro serotipos del virus del dengue en estudiantes de la Universidad Juárez Autónoma de Tabasco (UJAT) que acudieron a realizarse los análisis de sangre requeridos por la institución como parte del proceso de certificación de buena salud, durante los meses de septiembre a noviembre de 2005. Las muestras incluidas en el estudio fueron aquellas de las que se obtuvo tanto la firma de la carta de consentimiento informado, avalada por el comité de ética de la División Académica de Ciencias de la Salud (DACS/UJAT), así como la respuesta completa a la encuesta de caso para el dengue (Dirección General de Epidemiología, Secretaría de Salud de México); se excluyó del estudio a los estudiantes que al momento de la toma tuvieran algún signo o síntoma de enfermedad infecciosa evaluada por el médico del centro clínico de la DACS/UJAT. Las muestras se procesaron para la determinación de IgG contra el virus del dengue mediante el equipo ELISA-Dengue IgG Capture (Panbio) de acuerdo con las instrucciones del fabricante; los sueros positivos a IgG se seleccionaron entonces para realizar la prueba de neutralización en placa contra los cuatro serotipos del virus de acuerdo con el protocolo estandarizado del Instituto Pedro Kuri (La Habana, Cuba), para lo cual se usaron suspensiones virales de las cepas de DENV-1 (Hawai), DENV-2 (NGC), DENV-3 (H87) y DENV-4 (H241). Para cada ensayo se emplearon 50 unidades formadoras de placa por muestra y los resultados se expresaron en términos de la dilución a la cual se obtuvo $50 \%$ de reducción del número de placas. Como control negativo se utilizó una mezcla de sueros negativos a IgG contra el virus del dengue obtenido de muestras incluidas en este estudio y como control positivo se utilizaron anticuerpos monoclonales neutralizantes específicos de serotipo (donados por G. Clark, CDC, Laboratorios San Juan, San Juan, Puerto Rico).

\section{Resultados}

De un total de 824 alumnos que acudieron a realizarse los estudios de certificación de buena salud, 273 (33.1\%) aceptaron participar en el estudio y llenaron el cuestionario; la mediana de edad de los participantes fue de 19 años (intervalo de 18 a 39 años), 210 (77\%) fueron mujeres y 266 alumnos (96\%) refirieron no haber padecido dengue o ignorarlo. 
La prevalencia de anticuerpos IgG contra el virus del dengue para esta población fue de 9.1\% (25/273); las características de esta población se presentan en el cuadro I y en general reflejan la misma distribución por género y edad que la población total; seis de ellos (24\%) reconocieron antecedentes de dengue y la gran mayoría señaló que había vivido en Tabasco toda su vida. El ensayo de reducción de placa lítica muestra que, a una dilución de 1:10 del suero (esta dilución se utilizó como límite para considerar un suero positivo), todos los sueros positivos para IgG contra el dengue neutralizan al DENV-2 (25/25), 68\% tuvo anticuerpos neutralizantes contra DENV-4 (17/25), 20\% los presentó contra el DENV-1 (5/25) y 4\% contra el DENV-3 (1/25). El cuadro II muestra los perfiles de reactividad por serotipo para cada suero; en total se encontró sólo $24 \%$ de respuesta homotípica con el DENV-2 (6/25); de la proporción restante, $60 \%(15 / 25)$ presenta respuesta heterotípica contra dos serotipos, sobre todo contra DENV-2 y DENV-4, y $16 \%(4 / 25)$ contra DENV-1, DENV-2 y DENV-4. Para representar la magnitud de la respuesta neutralizante los sueros se titularon; los sueros con títulos de anti-

Cuadro I

Características demográficas de los participantes CON MUESTRAS POSITIVAS A IGG DE DENGUE MEDIANTE ElisA de captura.Villahermosa, Tabasco, México, SEPTIEMBRE-NOVIEMBRE DE 2005

Número de muestras $(n=25) \quad \%$

\begin{tabular}{lcc} 
Masculino & 7 & 28 \\
\hline Femenino & 18 & 72 \\
\hline Edad & $19(18 \text { a } 30)^{*}$
\end{tabular}

Lugar nacimiento

\begin{tabular}{lrr} 
En los municipios de residencia & 23 & 92 \\
\hline Fuera de los municipios de residencia & 2 & 8
\end{tabular}

Residencia (municipio)

\begin{tabular}{llc} 
Cárdenas & 7 & 28 \\
\hline Centla & 3 & 12 \\
\hline Centro & 8 & 32 \\
\hline Cunduacan & 3 & 12 \\
\hline Macuspana & 3 & 12 \\
\hline Teapa & 1 & 4
\end{tabular}

Dengue

\begin{tabular}{lrr} 
Sí & 6 & 24 \\
\hline No & 19 & 76
\end{tabular}

*Mediana de la muestra (intervalo).
Cuadro II

Perfiles de anticuerpos neutralizantes

DE LOS SEROTIPOS DEL VIRUS DEL DENGUE MEDIANTE ENSAYO de REDUCCIÓN EN PLACA. Villahermosa, TABasco, MÉXICO, SEPTIEMBRE-NOVIEMBRE DE 2005

\begin{tabular}{|c|c|c|c|c|}
\hline Suero & Dil I:10* & Dil $>1: 10^{\ddagger}$ & Género & Dengue ${ }^{\S}$ \\
\hline E058 & D2 & D2 (>I:160) & $\mathrm{F}$ & $\mathrm{NO} / \mathrm{NS}^{\#}$ \\
\hline E078 & D2 & D2 (>I:I60) & $\mathrm{F}$ & NO/NS \\
\hline E019 & D2 & D2 (>I:160) & $M$ & sí \\
\hline E090 & D2 & D2 (>I:I60) & $\mathrm{F}$ & NO/NS \\
\hline E032 & D2 & D2 $(1: 80)$ & $\mathrm{F}$ & NO/NS \\
\hline E05I & D2 & D2 (>I:I60) & $\mathrm{F}$ & NO/NS \\
\hline E044 & D2,DI & D2 (I:80) & $\mathrm{F}$ & sí \\
\hline El44 & D2,D3 & D2 (>I:I60) & $\mathrm{F}$ & NO/NS \\
\hline J010 & D2,D4 & D2 $(1: 80)$ & $\mathrm{F}$ & NO/NS \\
\hline BI03 & D2,D4 & D2 (I:80), D4 (I:40) & $M$ & NO/NS \\
\hline D030 & D2,D4 & D2 (I:80), D4 (I:40) & M & NO/NS \\
\hline E033 & D2,D4 & D2 (>I:I60), D4 (I:20) & $\mathrm{F}$ & NO/NS \\
\hline E08I & D2,D4 & D2 (>I:I60), D4 (I:40) & M & sí \\
\hline E076 & D2,D4 & D2 (>I:I60), D4( I:80) & $\mathrm{F}$ & sí \\
\hline EI58 & D2,D4 & D2 (I:80), D4 (I:40) & $\mathrm{F}$ & NO/NS \\
\hline El62 & D2,D4 & D2 (I:80), D4( I:20) & $\mathrm{F}$ & NO/NS \\
\hline E088 & D2,D4 & D2 (>I:I60), D4 (I:40) & $\mathrm{F}$ & sí \\
\hline E020 & D2,D4 & D2 (>I:I60), D4 (I:80) & $\mathrm{F}$ & NO/NS \\
\hline E066 & D2,D4 & D2 (I:80),D4 (I:20) & M & NO/NS \\
\hline E00I & D2,D4 & D2 (>I:I60), D4 (I:40) & $\mathrm{F}$ & sí \\
\hline E053 & D2,D4 & D2 (I:80), D4 (I:20) & $\mathrm{F}$ & NO/NS \\
\hline J003 & D2,D4 & D2 (I:80), D4 (I:40) & M & NO/NS \\
\hline E0I7 & D2,D4,DI & D2 (I:80),D4 (I:40) & $\mathrm{F}$ & NO/NS \\
\hline E007 & D2,D4,DI & D2 (I:80), D4 (I:40) & $\mathrm{F}$ & NO/NS \\
\hline E056 & D2,D4,DI & D2 (>I:I60), D4 (I:40), DI (I:40) & $\mathrm{F}$ & $\mathrm{NO} / \mathrm{NS}$ \\
\hline
\end{tabular}

* Reducción de 50\% de las placas líticas de virus por serotipo (DI, D2, D3, D4) a la dilución sérica de I: 10

₹ Reducción de $50 \%$ de las placas líticas de virus por serotipo a diluciones séricas mayores de I: 10; la dilución se encuentra entre paréntesis al lado del serotipo

$\S$ Respuesta a la pregunta "EEl encuestado sabía si había padecido dengue?"

\# NO/NS= No/No sabe

cuerpos neutralizantes mayores de 1:10 para DENV-2 fueron la totalidad (25/25), para el DENV-4 disminuyó ligeramente a $64 \%$ (16/25), mientras que para el DENV-1 hasta $4 \%(1 / 25)$ y no se detectaron anticuerpos contra el DENV-3 en diluciones mayores de 1:10. La proporción de respuesta homotípica neutralizante se mantuvo en la misma magnitud, lo mismo que la respuesta heterotípica contra dos serotipos, DENV-2 y DENV-4, mientras que la respuesta heterotípica contra tres serotipos bajó de manera significativa de 16 a $4 \%(1 / 25)$. 


\section{Discusión}

La transmisión del dengue en el estado de Tabasco es muy particular; esto se debe a que, pese a hallarse en una región con las condiciones ecológicas más propicias para el desarrollo del mosquito vector, Aedes aegypti, y tener fronteras con dos de los estados con mayor transmisión informada, Veracruz y Chiapas, puede considerarse un estado de baja transmisión. ${ }^{9}$ Quizás por ello no resulta inesperado el contrastante entre la baja seroprevalencia de anticuerpos IgG identificada en este estudio (menor de $10 \%$ ) si se compara con los estudios de Veracruz y Yucatán (mayor de 70\%). ${ }^{7,8}$ Más aún, esta diferencia probablemente se explica por el diseño del estudio; en los casos referidos se consideraron para el diseño todos los grupos de edad y el nivel socioeconómico, mientras que en este estudio la selección de la población fue estricta, tanto por la edad como por el nivel socioeconómico. De manera adicional, en el caso específico del estudio de Yucatán, el protocolo se llevó a cabo mediante una técnica analítica $(\mathrm{IH})$ diferente de la utilizada en esta investigación. Al margen de que lo anterior sugiera que hay un subregistro de casos, llama la atención que en este estudio se reconociera una seroprevalencia de anticuerpos neutralizantes contra el DENV-3 de apenas 4\%, ya que durante 1997 este estado notificó cerca de 8300 casos y de 119 aislamientos virales de este brote 112 fueron del serotipo 3 (94\%). ${ }^{9}$ Además, esta población de estudiantes declaró haber vivido en el estado al menos por 15 años antes de la toma de la muestra y, asimismo, casi todos tuvieron sus domicilios de residencia en municipios donde fue más intensa la transmisión en 1997. Se cree que la reacción inmunitaria neutralizante específica de serotipo se observa por toda la vida, así que sólo hay dos explicaciones posibles. La más simple aduce que los anticuerpos neutralizantes de los sujetos no reconocen la cepa del virus utilizado para efectuar el ensayo de neutralización en placa ; existe evidencia empírica que apoya esta explicación (Sección de Dengue, Departamento de Virología, Instituto de Diagnóstico y Referencia Epidemiológica).Por otro lado, es posible que en este caso particular la magnitud del brote de 1997 se sobreestimara, dado que antes del año 2000 la notificación de casos no exigía una prueba confirmatoria de IgM contra el virus del dengue.

De acuerdo con la teoría llamada del "pecado original antigénico", la respuesta neutralizante contra el serotipo que infectó por primera vez al individuo siempre será la de mayor magnitud, cualquiera que sea la secuencia de infecciones subsecuentes; ${ }^{10}$ en consecuencia, parece claro que en la población de estudio, la infección por el DENV-2 es la más prevalente y que este serotipo es el que se transmite con mayor intensidad en esta población.
Por otro lado, resulta intrigante la reacción tan extendida contra el DENV-4 (60\%), dado que es un serotipo de muy baja transmisión en México y en Tabasco sólo se ha aislado en 1997 (3 de 119) y 2004 (1 de 9) ${ }_{;}^{9}$ se ha propuesto un efecto de supresión de la transmisión del DENV-4 por la circulación de los otros serotipos debido a la generación de inmunidad neutralizante cruzada, ${ }^{11}$ de tal modo que es probable que la elevada prevalencia de anticuerpos neutralizantes contra DENV-4 observada en este trabajo sea el resultado de la infección por DENV-2, en cuyo caso ésta sería la primera observación independiente que apoyara dicha hipótesis. Es poco probable que esta inmunidad neutralizante contra DENV-4 se explique por un subregistro en la transmisión, toda vez que en el periodo comprendido entre 1997 y 2004 se notificó el aislamiento de al menos 24 cepas de virus y ninguna de ellas resultó ser DENV-4. ${ }^{9}$ Es mucho más probable que los casos en los que se reconoció inmunidad heterotípica contra más de dos serotipos reflejen la circulación del DENV-1 porque este serotipo fue el segundo más aislado en 2005; aunque sólo la cuarta parte de los positivos a IgG contra el virus del dengue reconoció antecedentes de dengue (cuadro II), es posible que el resto se infectara de forma asintomática, pues la proporción de asintomáticos puede ser de $80 \%$ del total de infectados. ${ }^{12}$

Este tipo de estudios contribuye a conocer los determinantes del ser humano que influyen en la transmisión; si bien son modestos en términos del diseño epidemiológico, permiten conocer algunos elementos que orienten a estudios de mayor envergadura y que son indispensables para elaborar sistemas de vigilancia racionales y efectivos.

\section{Agradecimientos}

Los autores de este trabajo agradecen al Dr. Carlos Jesús Conde González la revisión crítica de la versión preliminar de este manuscrito. Miguel Angel López Alvarado participó en este trabajo con apoyo del Banco Santander Serfin (Convenio 010/06-UJAT-BECARIO-MOVILIDAD ESTUDIANTIL). Esta investigación fue parcialmente financiada con fondos del proyecto FOSISS SALUD-2005 -01-13852 otorgado a José Ramos Castañeda.

En memoria del Dr. Juan Ruiz Gómez.

\section{Referencias}

I. Kroeger A, Nathan M, Hombach J. Dengue. Nat Rev Microbiol 2004;2(5):360-36I.

2. Ligon BL. Dengue fever and dengue hemorrhagic fever: a review of the history, transmission, treatment, and prevention. Semin Pediatr Infect Dis 2005; I6(I):60-65. 
3. Anderson KB. Burden of symptomatic dengue infection in children at primary school in Thailand: a prospective study. Lancet 2007;369(957|): |452-1459.

4. Green S, Rothman A. Immunopathological mechanisms in dengue and dengue hemorrhagic fever. Curr Opin Infect Dis 2006; I9(5):429-436. 5. Wearing HJ, Rohani P. Ecological and immunological determinants of dengue epidemics. Proc Natl Acad Sci USA 2006; I03(3 I): I I 802-I I 807. 6. Halstead SB. Dengue in the Americas and Southeast Asia: do they differ? Rev Panam Salud Publica 2006;20(6):407-4I5.

7. Navarrete-Espinosa J.Prevalence of dengue and leptospira antibodies in the state of Veracruz, Mexico. Salud Publica Mex 2006;48(3):220-228.

8. Lorono-Pino MA, Farfan-Ale JA, Rosado-Paredes EP,Arjona-Torres Al,

Flores-Flores LF, Manzano-Cabrera LA, et al. Prevalencia de anticuerpos contra el virus dengue en Yucatán, México, 1985. Rev Latinoam Microbiol 1989;31:259-262

9. SINAVE. Dirección General de Epidemiología. Secretaría de Salud.

[Consultado en septiembre de 2007]. Disponible en: http://www.dgepi. salud.gob.mx/sinave/index.htm.

10. Guzman MG. Neutralizing antibodies after infection with dengue I virus. Emerg Infect Dis 2007; I3(2):282-186.

I I.Adams B. Cross-protective immunity can account for the alternating epidemic pattern of dengue virus serotypes circulating in Bangkok. Proc Natl Acad Sci USA 2006; 103(38): I4234- I 4239.

12. Guzman MG. Epidemiologic studies on Dengue in Santiago de Cuba, 1997. Am J Epidemiol 2000; I 52(9):793-799; discussion 804. 